\title{
Correction to: Patterns of genetic partitioning and gene flow in the endangered San Bernardino kangaroo rat (Dipodomys merriami parvus) and implications for conservation management
}

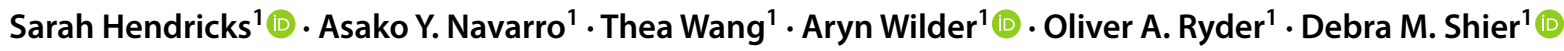

Published online: 28 July 2020

(c) Springer Nature B.V. 2020

\section{Correction to: Conservation Genetics \\ https://doi.org/10.1007/s10592-020-01289-z}

In the Original publication of the article, Table 2 was published incorrectly. The correct Table 2 is given in this Correction.

The original article has been corrected.

Publisher's Note Springer Nature remains neutral with regard to jurisdictional claims in published maps and institutional affiliations.

The original article can be found online at https://doi.org/10.1007/ s10592-020-01289-z.

Sarah Hendricks

shendri4@gmail.com

$\bowtie$ Debra M. Shier

dshier@sandiegozoo.org

1 San Diego Zoo Institute for Conservation Research, San Diego Zoo Global, 15600 San Pasqual Valley Road,

Escondido, CA 92027, USA 
Table 2 Summary statistics from microsatellite data among the range-wide sites: sample size $(\mathrm{N})$, allelic richness (AR), private allelic richness (PAR), observed heterozygosity $\left(\mathrm{H}_{\mathrm{O}}\right)$, expected het-

\begin{tabular}{|c|c|c|c|c|c|c|c|c|c|}
\hline Population & Site & $\mathrm{N}$ & $\mathrm{AR}$ & PAR & Ho & $\mathrm{He}$ & $\mathrm{F}$ & $\mathrm{Ne}(95 \% \mathrm{CI})$ & wang(xy) \\
\hline \multirow[t]{6}{*}{ Lytle Creek and Cajon Creek } & - & 51 & 10.86 & 2.57 & 0.78 & 0.8 & 0.043 & $85.8(67.7-114.2)$ & 0.01 \\
\hline & Hwy210, Lytle and Cajon Creeks & 3 & 3.64 & 0 & 0.76 & 0.62 & - & $-2.9(-3.3$-infinity $)$ & 0.00 \\
\hline & Glen Helen, Cajon Creek & 7 & 5.5 & 0.07 & 0.85 & 0.72 & - & - 164.5 (44.3-infinity) & 0.00 \\
\hline & Institution, Cajon Creek & 1 & 1.86 & 0.07 & 0.86 & 0.43 & - & $-0.3(-0.3-$ infinity $)$ & - \\
\hline & Lytle Creek Bank, Lytle Creek & 19 & 7.07 & 0 & 0.76 & 0.74 & 0.039 & $34.8(25.7-51.2)$ & 0.05 \\
\hline & Cajon Creek Bank, Cajon Creek & 21 & 9.5 & 0.43 & 0.77 & 0.81 & 0.069 & $130.5(76-400.7)$ & 0.01 \\
\hline \multirow[t]{5}{*}{ San Jacinto River } & - & 61 & 5.57 & 0.57 & 0.58 & 0.62 & 0.067 & $14.7(10.9-19.6)$ & 0.06 \\
\hline & Lake Park Dr. & 5 & 2.64 & 0 & 0.49 & 0.45 & 0.065 & $2.1(1.4-5.9)$ & 0.20 \\
\hline & San Jacinto & 22 & 3.93 & 0 & 0.52 & 0.54 & 0.095 & $31.1(17.3-84.1)$ & 0.11 \\
\hline & Hemet & 19 & 3.79 & 0.14 & 0.54 & 0.55 & 0.112 & $11(7.3-17.2)$ & 0.16 \\
\hline & Valle Vista & 15 & 4.5 & 0.21 & 0.74 & 0.64 & 0.003 & $39.4(19.5-235.7)$ & 0.03 \\
\hline \multirow[t]{4}{*}{ Santa Ana River } & - & 57 & 9.29 & 1.21 & 0.74 & 0.78 & 0.075 & $30.4(26.2-35.7)$ & 0.04 \\
\hline & Redlands & 22 & 5.64 & 0.14 & 0.73 & 0.7 & 0.057 & $9.1(7.3-11.5)$ & 0.16 \\
\hline & Mountain View & 15 & 6.14 & 0.14 & 0.72 & 0.69 & 0.088 & $13.9(10.8-18.3)$ & 0.17 \\
\hline & SBV \& Cone Camp & 20 & 8.07 & 0.71 & 0.78 & 0.77 & 0.061 & $60.9(42.6-101.7)$ & 0.03 \\
\hline
\end{tabular}

erozygosity $\left(\mathrm{H}_{\mathrm{E}}\right)$, weighted inbreeding coefficients $\left(\mathrm{F}_{\mathrm{IS}}\right)$ along with its associated $\mathrm{p}$ value, and effective population size $\left(\mathrm{N}_{\mathrm{E}}\right)$ with $95 \% \mathrm{CI}$ 